\title{
P-174
}

\section{Pharmacological Effects of Simple Medicinal Herbs Used in Persian Traditional Medicine for Treating Tremor}

\author{
Arman Zargaran ${ }^{*}$ and Mohammad M. Zarshenas
}

Department of Traditional Pharmacy, Faculty of Pharmacy, Shiraz University of Medical Sciences, Shiraz, Iran; and Shiraz University of Medical Sciences, Shiraz,Iran; E-mail: zargarana@sums.ac.ir

Management of tremor is a challenge for physicians. In addition to medication, surgical procedures such as deep brain stimulation (DBS) are performed in intractable cases. For some types of tremor, such as essential tremor, there are still no reliable treatment options. On the other hand, Complementary and traditional medicines have been used to treat tremor for centuries in many cultures (Frey \& Meyers, 2010). Traditional Persian Medicine (TPM) in the medieval period was one of the most well developed systems of medicine. In this regard, we investigated in some important Persian traditional manuscripts including the Canon of Avicenna (Avicenna, 1025AD), Ferdos-al-Hekmah of Tabari (Rabben Tabari, 848AD), Mansuri-fi-Teb of Rhazes (Rhazes, 906AD), AlAghraz-al-Tebbieh of Jorjani (Jorjani, 1131), Eksir-e-Azam of Azam Khan (Azam Khan, 1810AD), and Makhzan-al-Advieh of Aghili (Aghili Shirazi, 1771AD) to find simple medicines used to treat tremor. The identification of medicinal herbs is discussed in Kitab al-saydala fi Teb of Biruni (Biruni, 1050AD and Al-Havi of Rhazes (Rhazes, $10^{\text {th }}$ century). As result, 20 medicinal herbs and natural product were identified. In the next step, their pharmacological effects were studied to find the probable mechanisms of effect. It should be noted that many of mentioned medicinal herbs in medieval Persia have shown anti oxidant, dopaminergic and antimuscarinic properties. It can be the key role for their anti tremor activities. By this study many traditional natural products are suggested to further investigations for management of tremor. 